\title{
Magnetoacoustic tomography with current injection
}

\author{
LIU GuoQiang $^{1 *}$, HUANG Xin $^{1,2}$, XIA Hui $^{1} \&$ WU ShiZeng ${ }^{1}$ \\ ${ }^{1}$ Institute of Electrical Engineering, Chinese Academy of Sciences, Beijing 100190, China; \\ ${ }^{2}$ University of Chinese Academy of Sciences, Beijing 100049, China
}

Received February 28, 2013; accepted May 27, 2013; published online July 10, 2013

\begin{abstract}
Magnetoacoustic tomography combines the advantages of electrical impedance tomography with sonography. It can reconstruct conductivity images of biological tissue with high spatial resolution and high contrast. In addition, magnetoacoustic tomography is a new nondestructive functional imaging technology, with which we can realize the early diagnosis of human diseases. In this paper, through theory analysis we deduced the analytical formula of acoustic pressure. Computer simulation and experimental studies on magnetoacoustic tomography with current injection (MAT-CI) were made, and a copper ring was used as the model for the simulation and experiment. Experimental setup and methods are introduced, and the acoustic signals and the image of a metal object by means of MAT-CI are presented. Results of the simulation and experiment were in a good agreement. The reconstructed image of an acoustic source can reflect the conductivity information of a sample. The conductivity distribution of a sample can be obtained by means of MAT-CI. The results provide foundations of study on a model with complex conductivity distribution and tissues for MAT-CI.
\end{abstract}

magnetoacoustic tomography, reconstruction of acoustic source, applied current, convolution

Citation: Liu G Q, Huang X, Xia H, et al. Magnetoacoustic tomography with current injection. Chin Sci Bull, 2013, 58: 3600-3606, doi: 10.1007/s11434013-5964-2

Medical imaging creates and displays tissue lesions directly, and plays an important role in medical diagnosis. X-ray imaging technology [1] and X-ray computed tomography (CT) imaging technology [2] can cause damage to the human body because of their capability to ionize during the $\mathrm{X}$-ray scan. The ultrasonic imaging technique [3] provides higher-resolution images, and although the contrast of this technique is relatively low, although it is not harmful. Magnetic resonance imaging (MRI) [4] is a noninvasive method for body composition analysis because this technique does not use ionizing radiation (unlike CT scans and X-rays) $[5,6]$. However, the use of MRI is limited due to the high cost of equipment. All of the above medical imaging technologies use morphology and structure imaging, and their application is only focused on diagnosis of tissue or organ morphological structure that has been diseased, and cannot realize the early diagnosis of human diseases.

*Corresponding author (email: gqliu@mail.iee.ac.cn)
Electrical impedance tomography (EIT) [7] is a novel medical imaging technology. It has the outstanding advantages of noninvasive, functional imaging and medical image monitoring. As a functional imaging modality, EIT is very suitable for early diagnosis, and it has been used in various clinical applications and continues to attract the interest of substantial researcher, because of its functional imaging characteristics. However, the spatial resolution of EIT is low.

Magneto-acoustic tomography (MAT) [8] was proposed in recent years, which can combine ultrasound tomography with electrical impedance tomography. MAT can achieve noninvasive electrical impedance imaging with high spatial resolution. In MAT, the sample is placed in a static magnetic field, and a time-varying magnetic field is added around the sample. The time-varying magnetic field induces an eddy current in the sample or a time-varying current is injected into the sample directly. According to Lorentz's law, the current in the static magnetic field causes vibrations 
due to the Lorentz force and emits ultrasound signals. These ultrasound signs are collected by transducers located around the sample for reconstruction of a conductivity image of the sample, which reflects the distribution of conductivity [8]. Magnetoacoustic tomography combines the advantages of electrical impedance tomography with sonography fully and achieves functional imaging with high resolution and high contrast.

Compared with the other similar imaging modalities aimed at obtaining electrical impedance distribution such as EIT and magnetic resonance electrical impedance tomography (MREIT), MAT has unique features as follows [9]: in MAT, the measurement is of an acoustic signal instead of a magnetic field or voltage, so the effects that come from external electromagnetic interference is relatively little and the error is reduced to a certain extent. At the same time, it avoids the need of contact measurements and achieves high spatial resolution electrical impedance imaging. MAT is compatible with the MRI setup. In both imaging modalities, the sample is located in a static magnetic field. However, MAT is much less demanding in terms of field homogeneity and stability than MRI [9].

MAT includes magneto-acoustic tomography with magnetic induction (MAT-MI) [10-13] and magneto-acoustic tomography with current injection (MAT-CI). MAT-MI uses magnetic induction to induce eddy currents in the conductive sample and generates acoustic vibrations through the Lorentz force coupling mechanism, which was proposed by He group [9] in 2005. After that, a number of research units at home and abroad have carried out a series of studies on MAT-MI. He group [13] derived the formulae for the forward problem and the inverse problem, and studied conductivity reconstruction of MAT-MI [14]. Experimental studies were also carried out on copper loops [9], gel phantoms [11] and salted tissue samples [13]. Research at the Institute of Electrical Engineering, Chinese Academy of Sciences published their findings on the mechanism of sound source and to propose three methods to avoid the singularity of the divergence of Lorentz force on the interface between two mediums [15-17]. Experimental setup and methods were also established [18].

In MAT-CI, the ultrasound caused by the injected current in the conductive sample [19] is measured by transducers of the imaging system, so the realization of the excitation source is easier than MAT-MI. Currently, related research on MAT-CI is relatively less. The Institute of Biomedical Engineering, Chinese Academy of Medical Sciences [20] and Institute of Electrical Engineering, Chinese Academy of Sciences [21] carried out research on MAT-CI, the results of which proved the feasibility of the proposed MAT-CI approach with no relevant reports published elsewhere. The effect on pressure signals caused by ultrasonic transducer characteristics has not yet been considered at present. For image reconstruction, only a 1-D model was reconstructed by a direct tracing point method; ultrasonic transducers cannot be positioned accurately because of the use of a highly simple experimental device.

Both MAT and MAT-CI make use of electrical-acoustic coupled by the Lorentz force, where electric currents are produced in the sample and acoustic signals surrounding the object are measured. In MAT-CI, electric currents are injected into the sample directly, so interference on the ultrasonic detection equipment and static magnetic field can be avoided, which is caused by pulsed magnetic field in MATMI. The cost of MAT-CI is low and the device is easier to realize. To solve the problems above, we investigated the acoustic signal based on acoustic transducer characteristics, and the acoustic source distribution of the 2-D model was reconstructed for the pressure on the circular detection surface by the time reversal method. In the experimental study, we demonstrated a 2-D system in which the detector scans around the object in a circle and is controlled by a single chip microcomputer. In this system, an acoustic signal can be received more accurately. Two permanent magnets were positioned $\sim 2 \mathrm{~cm}$ under and over the sample; the permanent magnets can create a uniform magnetic field and avoid electromagnetic interference caused by the electromagnet.

We established a sheet copper ring model to study the problems above. Both computer simulation and experiments were conducted on the current distribution, vibration of sample and acoustic wave propagation and transducer characteristics.

\section{Theory}

\subsection{Forward problem of MAT-CI}

The forward problem describes two major physical processes in the signal generation mechanism of MAT-CI, i.e. current conduction in the sample, and the processes of the Lorentz force inducing an acoustic signal and acoustic wave propagation. Figure 1 shows the conceptual framework of MAT$\mathrm{CI}$ imaging. The area with black dots is the static magnetic field, the direction of which is perpendicular to the paper outward, which is $\boldsymbol{B}_{0}=B_{0} z, z$ is the direction of static magnetic field, $B_{0}$ is the magnetic flux density. The gray area is the copper ring plate to be detected, the conductivity of which is $\sigma_{1}$. The ultrasonic transducers are located around the sample in a circle, and both the sample and the transducers are immersed in pure water $\left(\sigma_{0}=0\right)$ for better ultrasound coupling. The current is injected into the sample in A and $\mathrm{B}$ areas using the electrodes.

On the exterior boundary of the sample, the current density of A and B areas is $\boldsymbol{J}_{\phi}^{e}$, and on the rest boundary, the normal component of the current density is zero. Assume the magnetic vector potential in the sample to be $\boldsymbol{A}$, and the magnetic flux density in the sample can be expressed as $\boldsymbol{B}=\nabla \times \boldsymbol{A}$. The sample in Figure 1 is axisymmetric, so the control equation of MAT-CI can be described as follows: 


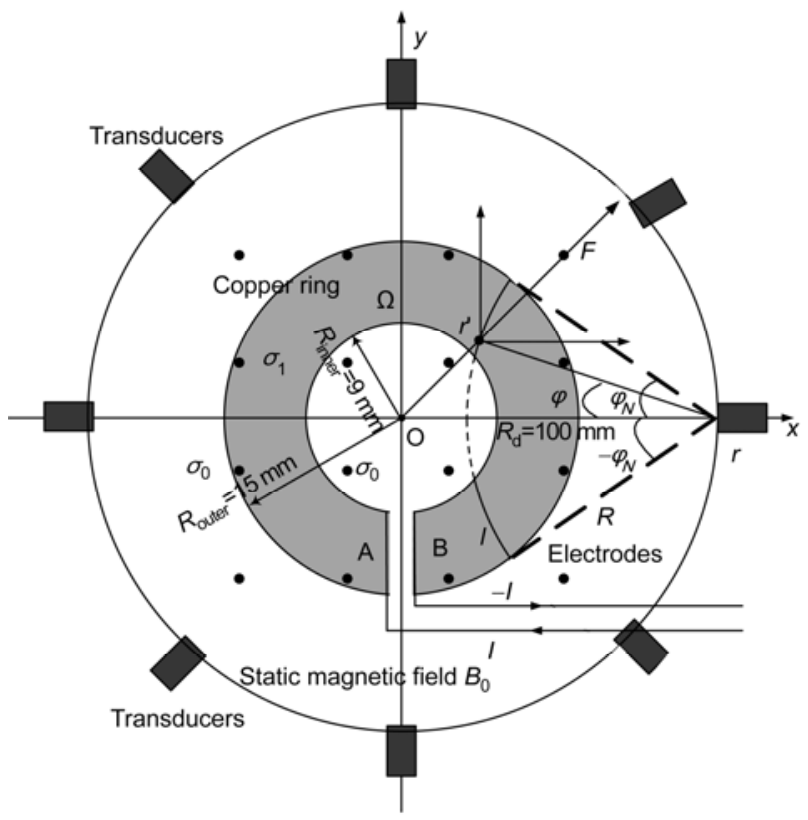

Figure 1 Illustration of the present MAT-CI system.

$$
\left\{\begin{array}{l}
\sigma \frac{\partial A}{\partial t}+\nabla \times\left(\frac{1}{\mu} \nabla \times \boldsymbol{A}\right)=J_{\phi}^{e}, \\
\boldsymbol{n} \times\left.\left(\frac{1}{\mu} \nabla \times \boldsymbol{A}\right)\right|_{\Gamma}=0,
\end{array}\right.
$$

where $\boldsymbol{n}$ is the normal component of the outer boundary, and $\Gamma$ is the outer boundary of the sample. The Lorentz force is excited by the current in the static magnetic field, and its expressions is as follows:

$$
\boldsymbol{f}\left(\boldsymbol{r}^{\prime}\right)=\boldsymbol{J}\left(\boldsymbol{r}^{\prime}\right) \times \boldsymbol{B}_{0},
$$

where $\boldsymbol{r}^{\prime}$ is the position of acoustic source and $\boldsymbol{J}\left(\boldsymbol{r}^{\prime}\right)$ is the total current density inside the sample.

In MAT-MC, the current in the sample causes particle vibrations and generates a detectable ultrasound signal. The acoustic signal is then measured by acoustic transducers around the object. The relation of acoustic pressure and distribution of the current density in the sample and static magnetic field is governed by the following wave equation [22]:

$$
\nabla^{2} p-\frac{1}{c_{s}^{2}} \frac{\partial^{2} p}{\partial t^{2}}=\nabla \cdot\left(\boldsymbol{J}\left(\boldsymbol{r}^{\prime}\right) \times \boldsymbol{B}_{0}\right),
$$

where $\nabla \cdot\left(\boldsymbol{J}\left(\boldsymbol{r}^{\prime}\right) \times \boldsymbol{B}_{0}\right)$ is the acoustic source term in the $\boldsymbol{r}^{\prime}$ position, $p$ is the acoustic pressure and $C_{s}$ is the acoustic speed in the medium, $\nabla^{2}$ is the Laplacian operator. The acoustic pressure can be obtained using Green's function [23]. If the focused transducer and very flimsy sample such as the sheet of copper using in this paper are used, the acoustic wave is measured only, which is emitted by the sample on the focal plane of the ultrasonic transducer. Thus, the integration can be calculated on a section of the sample. The section can be considered as an acoustic source, and the acoustic pressure at the position of the transducer can be derived as

$$
p(\boldsymbol{r}, t)=-\frac{1}{4 \pi} \nabla \cdot \int_{\Omega} \mathrm{d} \boldsymbol{r}^{\prime} \boldsymbol{J}\left(\boldsymbol{r}^{\prime}\right) \times \boldsymbol{B}_{0} \frac{\delta\left(t-R / c_{s}\right)}{R},
$$

where $\boldsymbol{r}$ is the position of transducers, $R=\left|\boldsymbol{r}^{\prime}-\boldsymbol{r}\right|$, and $\Omega$ is the whole area of integration that contains all acoustic sources. As is shown in eq. (4), at the moment $t$, the acoustic pressure $p(\boldsymbol{r}, t)$ collected at $\boldsymbol{r}$ is proportional to the integral results of all acoustic source terms $\boldsymbol{J}\left(\boldsymbol{r}^{\prime}\right) \times \boldsymbol{B}_{0}$ on the circle, the center of which is at the point $\boldsymbol{r}$ with a radius of $t c_{s}$. Making a further derivation on eq. (4), we obtained the formula of acoustic pressure at the point of the transducer:

$$
p(\boldsymbol{r}, t)=-\frac{1}{4 \pi} \nabla \cdot \int_{-\phi_{N}}^{\phi_{N}} \frac{\boldsymbol{f}(-R \cos \phi+r)}{\sqrt{R^{2}+r^{2}-2 R r \cos \phi}} \mathrm{d} \phi,
$$

where $r$ is the distance from point $\mathrm{O}$ to the position of transducer $\boldsymbol{r}, \phi_{N}$ and $-\phi_{N}$ are the upper and lower limits of integration, respectively (Figure 1). According to eq. (5), we can calculate the sound pressure value at the position of $\boldsymbol{r}$.

\subsection{Acoustic pressure collected by a transducer}

$h(t)$ is defined as the impulse response of the transducer. In MAT-CI, the resultant signal $w(\boldsymbol{r}, t)$ collected by the transducer is a convolution between the acoustic pressure $p(\boldsymbol{r}, t)$ and the impulse response $h(\boldsymbol{r}, t)$ of the transducer as [24]

$$
w(\boldsymbol{r}, t)=p(\boldsymbol{r}, t) \otimes h(t) .
$$

Eq. (7) can be expressed with their product in the frequency domain as

$$
W(\boldsymbol{r}, \omega)=P(\boldsymbol{r}, \omega) H(\omega),
$$

where $W(\boldsymbol{r}, \omega), P(\boldsymbol{r}, \omega)$ and $H(\omega)$ are the Fourier spectra of $w(\boldsymbol{r}, t), p(\boldsymbol{r}, t)$ and $h(t)$, respectively. Based on the least mean square error estimation, acoustic pressure $p(\boldsymbol{r}, t)$ at the position of the transducer can be restored with the Wiener filter for the deconvolution in the presence of noise [24],

$$
p(\boldsymbol{r}, t)=\mathrm{FFT}^{-1}\left(\frac{W(\boldsymbol{r}, \omega) H *(\omega)}{H(\omega) H *(\omega)+C}\right),
$$

here $\mathrm{FFT}^{-1}$ denotes the inverse Fourier transform operation, the asterisks "*" mean the complex conjugation and $C$ is an approximate constant based on the spectral density ratio of signal to noise. Using the resultant signal $w(\boldsymbol{r}, t)$ collected by the transducer we can obtain the acoustic pressure $p(\boldsymbol{r}, t)$ at the position of the transducer through deconvolution.

\subsection{Inverse problem of MAT-CI}

The inverse problem of MAT-CI is to study how to recon- 
struct the acoustic source terms (Lorenz divergence) distribution from the resultant signal $w(\boldsymbol{r}, t)$ that we can measure around the sample. During the image reconstruction process, if the resultant signal $w(\boldsymbol{r}, t)$ is only used, which is collected from one direction of the transducer, its numerical value cannot be identified precisely. However, if the resultant signals $w(\boldsymbol{r}, t)$ are used, which are collected from various directions of the transducers, a 2-D image can be reconstructed. The acoustic source term $\nabla \cdot\left(\boldsymbol{J}\left(r^{\prime}\right) \times \boldsymbol{B}_{0}\right)$ in eq. (3) can be obtained by the means of the simplified back projection algorithm for acoustic sources [25,26].

$$
\begin{aligned}
& \nabla \cdot\left(\boldsymbol{J}\left(r^{\prime}\right) \times \boldsymbol{B}_{0}\right) \\
& \approx \frac{1}{2 \pi c_{s}^{3}} \oint \mathrm{d} s \frac{\boldsymbol{n} \cdot\left(\boldsymbol{r}-\boldsymbol{r}^{\prime}\right)}{\left|\boldsymbol{r}-\boldsymbol{r}^{\prime}\right|^{2}} p^{\prime \prime}\left(\boldsymbol{r},\left|\boldsymbol{r}-\boldsymbol{r}^{\prime}\right| / c_{s}\right),
\end{aligned}
$$

where $S$ is the surface on which acoustic transducers are placed, $p^{\prime \prime}$ is the second derivative of the acoustic pressure over time, and $\boldsymbol{n}$ is the normal vector of $S$ at $\boldsymbol{r}^{\prime}$ position. Eq. (9) indicates that the Lorenz divergence distribution can be reconstructed from $p(\boldsymbol{r}, t)$ at the position of the transducer.

\section{Simulation study}

\subsection{Design of simulation model}

To verify the validity of the proposed theory of MAT-CI, 2-D simulations were conducted for a copper ring on the calculation of acoustic pressure $p(\boldsymbol{r}, t)$ at the position of the transducer, acoustic pressure collected by the transducer and the image reconstruction of ultrasonic source. The conductivity distribution of the copper ring with a thickness of $1 \mathrm{~mm}$ is shown in Figure 1. The outer and inner radius of the copper ring is 15 and $9 \mathrm{~mm}$ respectively. The copper and transducer were immersed into pure water of $\sigma_{0} \approx 0 \quad \mathrm{~S} / \mathrm{m}$ for acoustic coupling. The acoustic velocity in the water is $1500 \mathrm{~m} / \mathrm{s}$. The central frequency of the transducer is $1 \mathrm{MHz}$. The transducer scans around the sample in a circular orbit with a radius of $100 \mathrm{~mm}$. The acoustic system was considered to be acoustically homogeneous without the consideration of acoustic reflection, dispersion and attenuation.

\subsection{Numerical simulations of acoustic pressure}

During simulations, the resultant signal $w(\boldsymbol{r}, t)$ collected by the transducer and acoustic pressure $p(\boldsymbol{r}, t)$ at the position of the transducer were calculated based on eqs. (5) and (6) with a step angle of $2^{\circ}$. Because the copper ring is axisymmetric, the resultant signal $w(\boldsymbol{r}, t)$ collected by the transducer, is the same at every measured angle. Figure 2(a) shows the acoustic pressure $p(\boldsymbol{r}, t)$ obtained at an angle of $0^{\circ}$, where the acoustic pressure begins at $56.7 \mu$ s and ends at $76.7 \mu$ s. The moments 56.7 and $76.7 \mu$ s correspond to the propagation time, respectively, in which the acoustic pressure $p(\boldsymbol{r}, t)$ induced by the two outsides of the copper ring propagates to the transducer.

Figure 2(b) displays the resultant signal $w(\boldsymbol{r}, t)$ collected by the transducer on the basis of acoustic transducer characteristics $h(t)$. From Figure 2(b), the two wave clusters represent the two outer sides of the copper ring on the axis direction of the ultrasonic transducer.

\subsection{Simulation of image reconstruction}

Based on the back projection algorithm of eq. (9), the Lorentz force divergence image of MAT-CI was reconstructed as illustrated in Figure 3 with 180 simulated values of acoustic pressure $p(\boldsymbol{r}, t)$. The reconstructed image clearly displays the shape and size of the outer and inner media of the copper ring.

\section{Experimental}

\subsection{Experimental apparatus and procedure}

Figure 4 shows the diagram of the MAT-CI system setup. In this setup, pure water to a certain height was placed in the
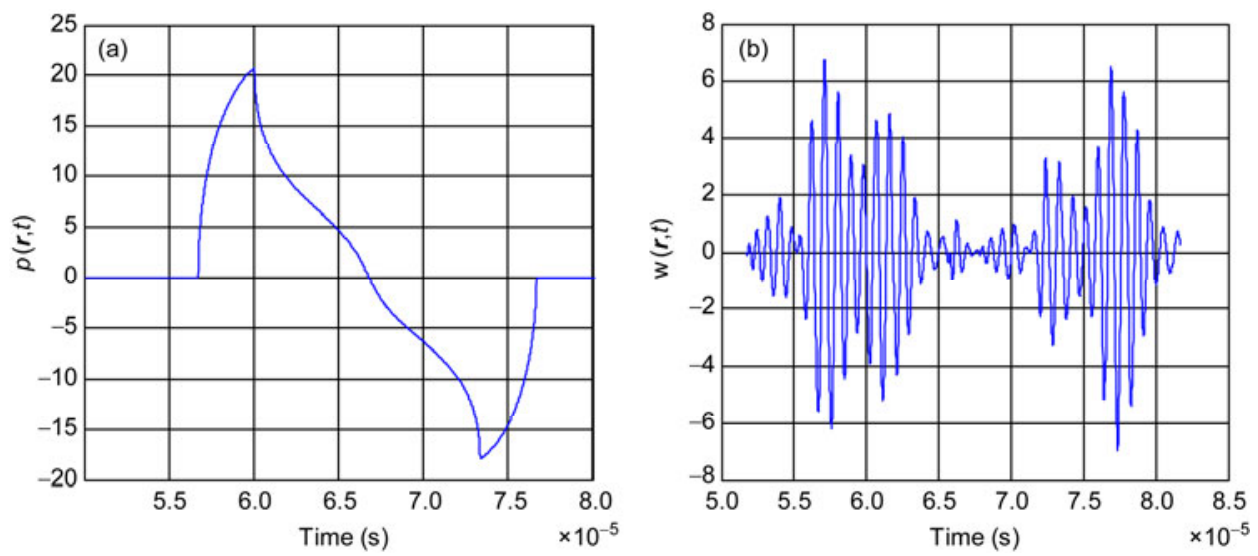

Figure 2 (Color online) (a) Acoustic pressure waveform $p(\boldsymbol{r}, t)$ at the transducer position. (b) The acoustic pressure waveform $w(\boldsymbol{r}, t)$ detected by the transducer. 


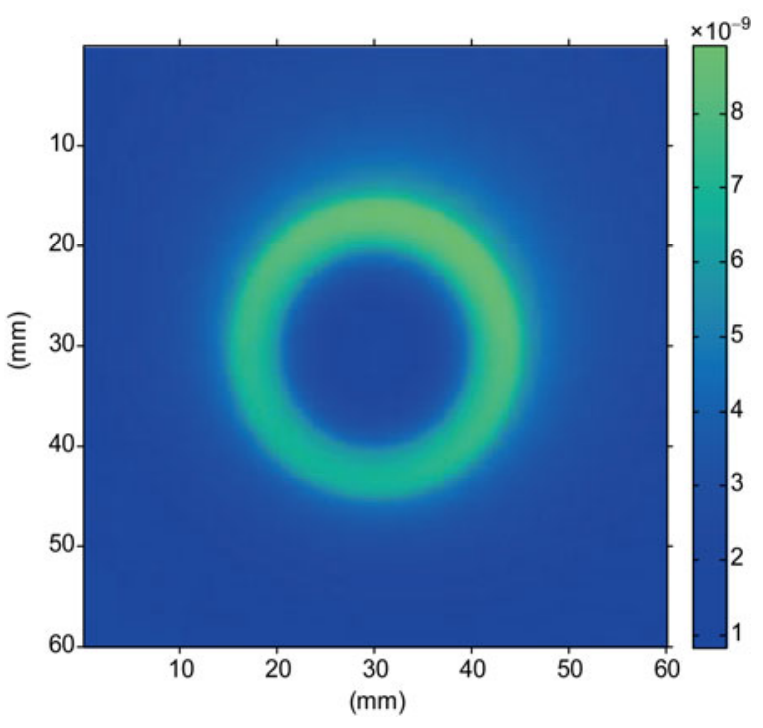

Figure 3 Reconstructed Lorentz force distribution image by the simulation data.

water tank. Both samples and the transducer were immersed in the water for good acoustic signal coupling. Two permanent magnets were used to get an approximately uniform magnetic field of $0.2 \mathrm{~T}$ between them. Current excitation consists of a signal generator and an amplifier, where the signal source adds a time-varying current to the sample through the power amplifier. The ultrasonic detection system is composed of ultrasonic transducers, an amplifier, an oscillograph, a stepper motor and a drive control system. The ultrasound transducer is driven by a stepper motor that is controlled by a microcomputer chip to scan around the sample in a circular orbit; acoustic signals were collected and recorded each time as long as the ultrasound transducer turned to a certain angle. The signal from the transducer was first amplified, and then displayed and recorded by the oscilloscope. Then, the collected signal was transferred to a computer for processing.

\subsection{Acoustic detection experiment}

A copper ring was used as the experimental sample as shown in Figure 5. The inner and outer radiuses of the copper ring are 9 and $15 \mathrm{~mm}$, respectively. During the experiment, the copper ring was placed on a bracket and located between the two permanent magnets to ensure that the sample and center axis of ultrasonic transducer (Panametrics V303, diameter, $19 \mathrm{~mm}$ ) are at the same height. The flux density of the magnetic field along the $z$-axis was $0.1 \mathrm{~T}$. The signal generator provided a square waveform signal with an amplifier amplitude of $10 \mathrm{~V}, 0.5 \%$ duty and a frequency of $2 \mathrm{kHz}$. The amplification of the amplifier was 20, the output current of which was 2 A. The ultrasonic transducer was installed on a mechanical scanning device, and was scanned around the object in a circular orbit with a radius of $100 \mathrm{~mm}$, the step size of the scanning is $2^{\circ}$. The signal from the transducer was first amplified, and then was sampled at $1 \mathrm{GHz}$ by a computer.

Figure 6 is a figure of acoustic signal waveform obtained from an oscillograph, where the horizontal axis represents time $(10 \mu \mathrm{s} / \mathrm{div})$; the vertical axis represents measured voltage, and the pulse pointed by the left arrow is a pulsed magnetic field caused by spatial coupling. $\mathrm{CH} 1$ is a waveform of the synchronous signal ( $1 \mathrm{~V} / \mathrm{div})$, the reference time point is the initial time signal of $\mathrm{CH} 1$, and $\mathrm{CH} 2$ is the waveform of the acoustic pressure $w(\boldsymbol{r}, t)(2 \mathrm{mV} / \mathrm{div})$ collected by the transducer. There are two acoustic pressure waveforms with a time delay of about $16 \mu \mathrm{s}$, which matches the distance between the front and rear boundary of the copper ring according to the position of the transducer. It shows that the two acoustic pressures correspond to the two outer boundaries. The acoustic signal waveform obtained by the simulation is similar to the waveform collected in the experiment.

\subsection{Image reconstruction of the experiment}

Figure 7 is the image of an acoustic source reconstructed by

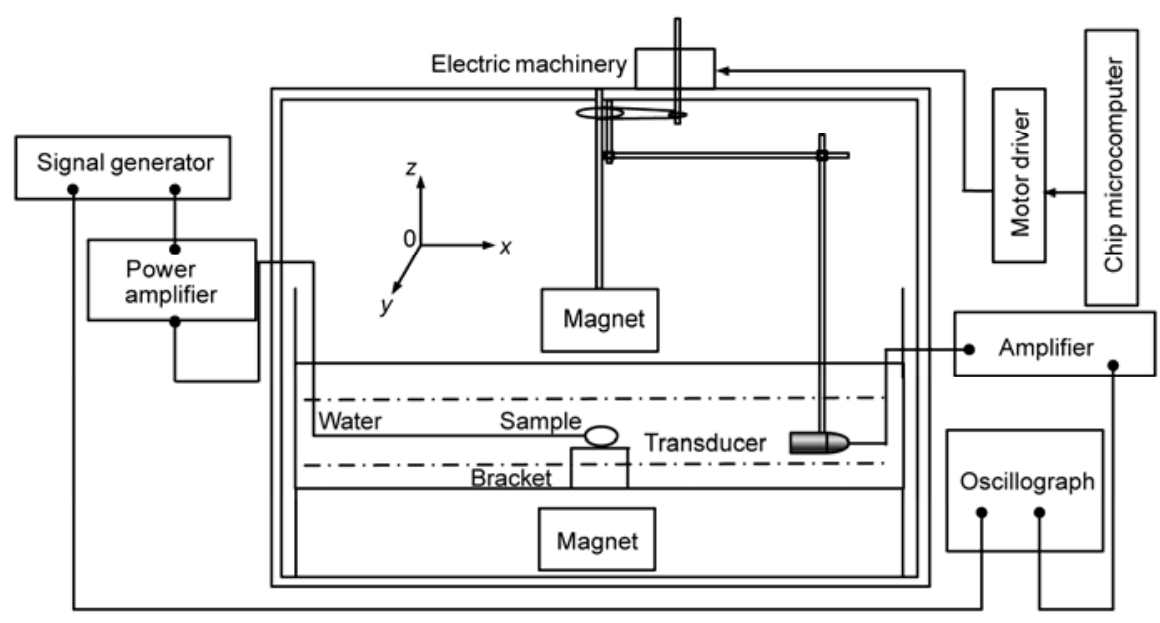

Figure 4 Experimental setup. 


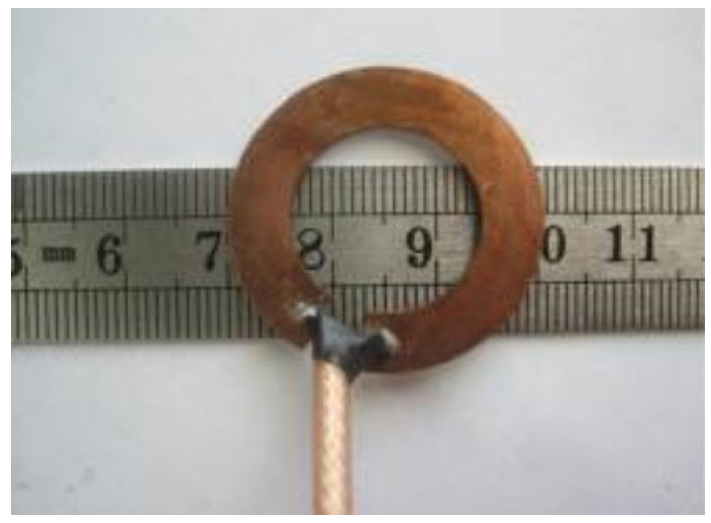

Figure 5 Photo of the copper ring.

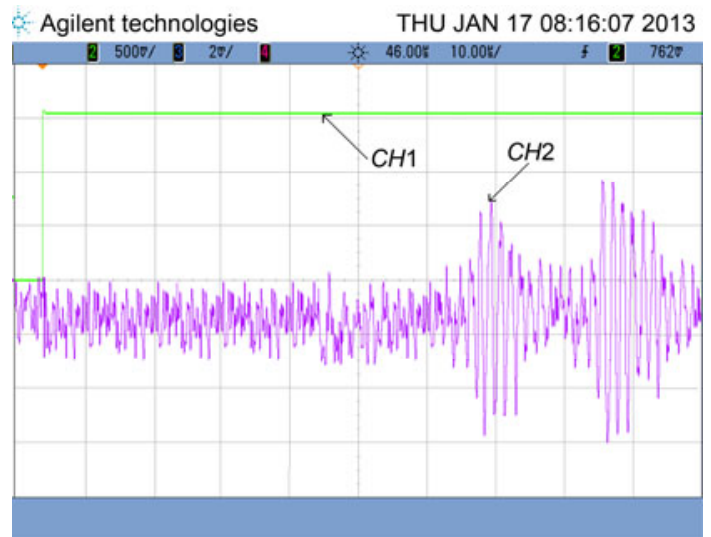

Figure 6 The signals from the copper ring.

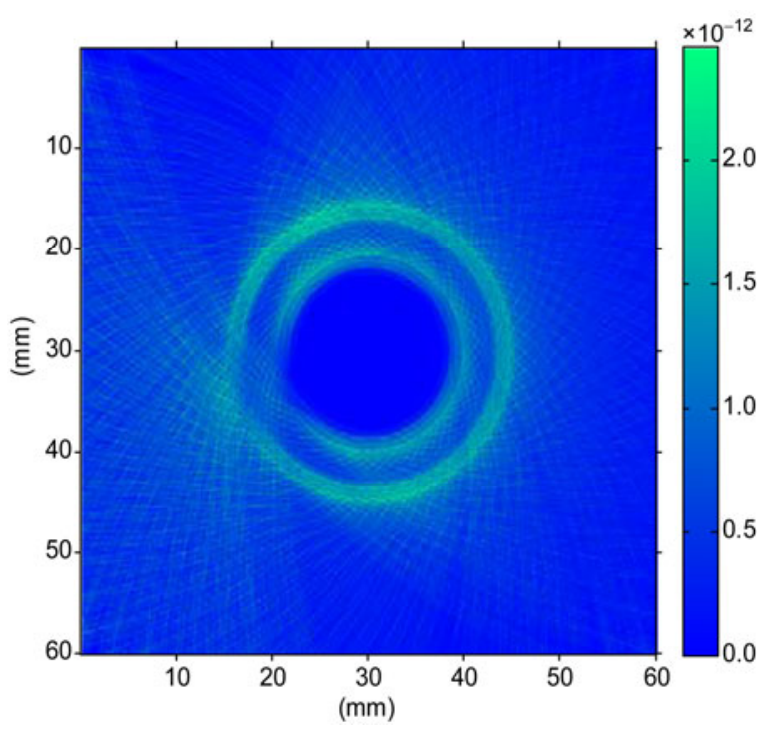

Figure 7 Reconstructed Lorentz force distribution image by the measured data.

an acoustic waveform measured in the experiment. The image agrees well with the shape and size of the copper ring. The current is high at each conductive boundary of the sample because of the skin effect, corresponding to the large
Lorenz force that leads to high vibration amplitude of the acoustic source. The reconstructed image represents the shape and size of the copper ring, and can reflect the position distribution of conductivity changes in the sample.

\section{Conclusion}

In the present paper, a study was performed on MAT-MC. In the theoretical section, we provided formulae for acoustic signals based on the theories of electromagnetic fields, sample vibration and ultrasonic detection. A computer simulation was conducted on a copper ring to obtain the acoustic pressure of $p(\boldsymbol{r}, t)$, and the acoustic image of MAT-CI was reconstructed based on a back projection algorithm. Experimental setup and methods were introduced, and the acoustic signals and the image of a copper ring were presented. Acoustic signal can be obtained more accurately using an acoustic transducer that scans around the object in a circle and controlled by single chip microcomputer. The results of the computer simulation were consistent with experimental results, and the reconstructed image of the copper ring was in good agreement with the shape of the sample. The results of present studies offer a new idea for reconstruction of the conductivity distribution, and provide a new method of imaging for early diagnosis of human tissue disease. It provides a theoretical basis and technical approach for the feasible application of MAT-CI in impedance imaging.

This work was supported by the National Natural Science Foundation of China (50977084, 2009B48, 61002036, 61271424 and 51137004).

1 Yang X P. The development and application of X-ray diffraction (in Chinese). J Shanxi Normal Univ: Nat Sci Ed, 2007, 21: 72-76

2 Wang G, Yu H Y, Brue D M. An outlook on X-ray CT research and development (in Chinese). Chin J Med Instrum, 2008, 32: 157-169

3 Yu J H, Wang Y Y. Simulation study of medical ultrasound imaging (in Chinese). Tech Acoust, 2011, 30: 33-40

4 Lauterbur P C. Image formation by induced local interaction: Examples employing nuclear magnetic resonance. Nature, 1973, 242: 190_ 191

5 Zhang S R, Ren J M, Pei F K. The research progress of MRI contrasts agents (in Chinese). Prog Chem, 1995, 7: 98-112

6 Qiu Z W, Pei F K. Spectroscopy of Nuclear Magnetic Resonsance (in Chinese). Beijing: Science Press, 1989. 533

7 Paulson K, Lionheart W, Pidecock M. Optimal experiments in electrical-impedance tomography. IEEE Trans Med Imaging, 1993, 12: 681-686

8 Jiang L T, Liu G Q, Wang H. Development of magnetoacoustic tomography (in Chinese). Int Biomed Eng, 2009, 32: 113-116

$9 \mathrm{Xu} \mathrm{Y,} \mathrm{He} \mathrm{B.} \mathrm{Magnetoacoustic} \mathrm{tomography} \mathrm{with} \mathrm{magnetic} \mathrm{induction}$ (MAT-MI). Phys Med Biol, 2005, 50: 5175-5187

$10 \mathrm{Hu} \mathrm{G}, \mathrm{He}$ B. Magnetoacoustic imaging of electrical conductivity of biological tissues at a spatial resolution better than $2 \mathrm{~mm}$. PLoS One, 2011, 6: 1-9

11 Li X, Xu Y, He B. Magnetoacoustic tomography with magnetic induction for imaging electrical impedance of biological tissue. J Appl Phys, 2006, 99: 066112 
12 He B. High-resolution functional source and impedance imaging. Proc Annu Int Conf IEEE-EMBS, 2005, 4178-4182

13 Li X, He B. Magnetoacoustic tomography with magnetic induction (MAT-MI) for electrical conductivity imaging. 31st Annual International Conference of the IEEE Engineering in Medicine and Biology Society, 2009. 3173-3176

14 Xia R M, Li X, He B. Magnetoacoustic tomographic imaging of electrical impedance with magnetic induction. Appl Phys Lett, 2007, 91: 083903

15 He W J, Liu G Q, Zhang Y. Research on forward problem of magnetoacoustic tomography with magnetic induction (I)-Sound field simulation method based on pressure-velocity coupling equation (in Chinese). Mod Sci Instrum, 2010, 1: 9-13

16 Liu G Q, He W J, Xia H. Research on forward problem of magnetoacoustic tomography with magnetic induction (II) - Sound field simulation method based on displacement equation (in Chinese). Mod Sci Instrum, 2010, 1: 14-16

17 Liu G Q, He W J, Xia H. Research on forward problem of magnetoacoustic tomography with magnetic induction (III)—Sound field simulation method based on the sound source of divergence of lorentz force optimized with weak form. Method based on displacement equation (in Chinese). Mod Sci Instrum, 2010, 1: 17-20

18 Huang X, Liu G Q, Xia H. Study of pulsed magnetic field used in MAT-MI (in Chinese). Trans China Electrotech Soc, 2013, 2: 67-72
19 Liu Z P, Yin T. Imaging with current exciting device based on magnetoacoustic effect (in Chinese). Chinese Patent, 201010585231, 2011-06-08

20 Zhang S Q, Yin T, Ma R. Study on imaging with current exciting conductivity model based on magnetoacoustic effect (in Chinese). Chin J Biomed Eng, 2011, 30: 801-806

21 Huang X, Liu G Q, Xia H. Study for power of magnetoacoustic tomography with injecting current by experiment (in Chinese). Adv Technol Electr Eng Energy, 2012, 31: 87-89

22 Jiang J Z, Qiu X J. Spatial distribution imaging of steady-state sound field with the ray-tracing method. J Nanjing Univ: Nat Sci, 2007, 1: 56-65

23 Li X, Xu Y, He B. Imaging electrical impedance from acoustic measurements by means of magnetoacoustic tomography with magnetic induction (MAT-MI). IEEE Trans Biomed Eng, 2007, 54: 323-330

24 Li Y L, Liu Z B, Ma Q Y. Two-dimensional lorentz force image reconstruction for magnetoacoustic tomography with magnetic induction. Chin Phys Lett, 2010, 27: 084302

$25 \mathrm{Xu} \mathrm{Y,} \mathrm{Wang} \mathrm{L} \mathrm{H} \mathrm{V.} \mathrm{Time} \mathrm{reversal} \mathrm{and} \mathrm{its} \mathrm{application} \mathrm{to} \mathrm{tomography}$ with diffracting sources. Phys Rev Lett, 2004, 92: 33902

26 Li X, Xu Y, He B. A Phantom study of magnetoacoustic tomography with magnetic induction (mat-mi) for imaging electrical impedance of biological tissue. J Appl Phys, 2006, 99: 066112

Open Access This article is distributed under the terms of the Creative Commons Attribution License which permits any use, distribution, and reproduction in any medium, provided the original author(s) and source are credited. 